\title{
Characterization of the spatial structure of local functional connectivity using multi-distance average correlation measures
}

Didac Macia, $\mathrm{MSc}^{1}$; Jesus Pujol, $\mathrm{MD}^{1,2^{*}}$; Laura Blanco-Hinojo, $\mathrm{PhD}^{1,2}$; Gerard Martínez-Vilavella, $\mathrm{MSc}^{1}$; Rocío Martín-Santos ${ }^{3}, \mathrm{MD}$; Joan Deus, $\mathrm{PhD}^{1,4}$

${ }^{1}$ MRI Research Unit, Department of Radiology, Hospital del Mar, Passeig Marítim 25-29, 08003, Barcelona, Spain

${ }^{2}$ Centro Investigación Biomédica en Red de Salud Mental, CIBERSAM G21, Dr. Aiguader 88, 08003, Barcelona, Spain

${ }^{3}$ Department of Psychiatry and Psychology, Hospital Clínic, Institut d’Investigació Biomédica August Pi I Sunyer, CIBERSAM G25, 08036, Barcelona, Spain

${ }^{4}$ Department of Clinical and Health Psychology, Autonomous University of Barcelona, 08193, Spain

Dídac Macià _ didac.macia@gmail.com

Jesus Pujol 21404jpn@comb.cat

Laura Blanco-Hinojo_ laura.blanco02@gmail.com

Gerard Martínez-Vilavella g.martinezvilavella@gmail.com

Rocío Martín-Santos RMSANTOS@clinic.cat

Joan Deus_ joan.deus@uab.cat

Running head: The spatial structure of functional connectivity

Keywords: brain activity, fMRI, local activity integration, connectivity maps

*Corresponding author: Dr. Jesus Pujol. MRI Department, Hospital del Mar, Passeig Marítim 25-

29. 08003, Barcelona, Spain. Email: 21404jpn@comb.cat Telephone: +34932212180 Fax: $+34932212181$ 


\begin{abstract}
There is ample evidence from basic research in neuroscience of the importance of local corticocortical networks. Millimetric resolution is achievable with current functional MRI (fMRI) scanners and sequences, and consequently a number of "local" activity similarity measures have been defined to describe patterns of segregation and integration at this spatial scale. We have introduced the use of Iso-Distant local Average Correlation (IDAC), easily defined as the average fMRI temporal correlation of a given voxel with other voxels placed at increasingly separated iso-distant intervals, to characterize the curve of local fMRI signal similarities. IDAC curves can be statistically compared using parametric multivariate statistics. Furthermore, by using RGB colorcoding to display jointly IDAC values belonging to three different distance lags, IDAC curves can also be displayed as multi-distance IDAC maps. We applied IDAC analysis to a sample of 41 subjects scanned under two different conditions, a resting state and an auditory-visual continuous stimulation. Multi-distance IDAC mapping was able to discriminate between gross anatomofunctional cortical areas and, moreover, was sensitive to modulation between the two brain conditions in areas known to activate and de-activate during audio-visual tasks. Unlike previous fMRI local similarity measures already in use, our approach draws special attention to the continuous smooth pattern of local functional connectivity.
\end{abstract}




\section{List of Abbreviations}

ACF: Autocorrelation Function

ASL: Arterial Spin Labelling

Cohe-ReHo: Coherence Regional Homogeneity

EPI: Echo-Planar Imaging

fMRI: Functional Magnetic Resonance Imaging

IDAC: Iso-Distant Average Correlation

KCC: Kendall's Coefficient of Concordance

MRI: Magnetic Resonance Imaging

ReHo: Regional Homogeneity 


\section{Introduction}

Local activity integration is one of the most important organizational principles of the brain in vertebrates (Abeles, 1982; Felleman and Van Essen, 2011; Tononi, 1994), prevalent at a neuron's scale as well as at larger scales (Burkhalter, 2016). Cortico-cortical axon collaterals are known to innervate neuronal column units stretching several millimeters away in the cortex mantle (Amir et al., 1993; Varela et al., 2001). Excitatory (or inhibitory) events do not occur in a spatially isolated manner; instead, they involve a regional neighborhood whose coupling gradually decays as recordings are taken farther away from the activity source (Grinvald et al, 2015; Liang et al, 2015) .

For a neuroimaging researcher, the question arises whether local activity coupling or local functional connectivity can be accessed via fMRI. Earlier fMRI studies have already demonstrated that it is possible to extract neurally significant results from local similarity measures and have applied them to hundreds of clinical studies. Coming from graph theory, Tomasi and Volkow (2010) and Sepulcre et al. (2010) proposed the use of local connectivity degree mapping, which basically counts the number of local temporal correlations above a certain significant threshold within a local neighborhood of a given voxel. Another popular local similarity measure proposed by Zang et al. (2004) is regional homogeneity (ReHo), which uses the Kendall's coefficient of concordance (KCC) (Baumgartner et al., 1999; Kendall and Gibbons, 1990), and, to a lesser degree, Cohe-Reho (Liu et al., 2010), which uses the frequency coherence to determine the temporal similarity of a given voxel with its adjacent voxels. The number of clinical publications indexed in the Pubmed database using ReHo is today greater than 200, a figure that will give the reader an idea of the growing interest in the use of local similarity measures. In such studies, local similarity measures have been used to characterize functional asymmetries of the human brain (Tomasi and Volkow, 2012a), the effects of task performance (Tomasi et al., 2014), gender differences (Xu et 
al., 2015), aging (Tomasi and Volkow, 2012b; Bernier et al., 2017), genetic disorders (Pujol et al., 2015), and, above all, neurological and psychiatric disorders (Liu et al., 2008; Tomasi and Volkow, 2012c; Pujol et al., 2014b).

Most local similarity measures use predefined neighborhood sizes, whether it is as an arbitrary distance setting in terms of a neighborhood radius in $\mathrm{mm}$ (Sepulcre et al., 2010), as a fixed number of adjacent voxels (Zang et al., 2004), or as a varying number of adjacent voxels determined by some kind of searching algorithm (Tomasi and Volkow, 2010). However, in all of them, the definition of what is local and what is not remains arbitrary and dychotomic. Our present work is precisely motivated by the notion that, by limiting the concept of locality to one "optimal" threshold distance, we risk losing the capacity of describing the rich smooth spatial gradient of local fMRI correlations.

To characterize such radial gradient voxelwise, we defined the concept of Iso-Distant Average Correlation (IDACs), which is the average temporal correlation of one voxel with all its neighboring voxels placed at different spatial lags. Several IDAC values computed from increasing distance lags generate IDAC curves, which we used extensively in this study. We hypothesized that IDAC curves would be able to capture the smooth spatial structure of functional connectivity with relation to distance.

IDAC values can easily be plotted, compared with multivariate statistics as Hotelling's $\mathrm{T}^{2}$ tests (Anderson, 2003; Worsley et al., 2004), and even represented as multi-distance brain maps (see Methods). We further hypothesized that IDAC values would be able to capture differences between adjacent histological areas as well as different brain functional states. Like other local similarity measures, IDAC would allow us to compare continuous activity conditions without the requirement of a changing (baseline-activation) paradigm. 
To test the sensitivity of IDAC, we applied it to a sample of 41 subjects during an eyes-closed resting state versus an auditory-visual continuous presentation. We used the resting state to assess the tissue specificity of the measure by generating group-average multi-distance IDAC maps, thanks to the display strategy of RGB color overlays that allowed us to visualize several distances jointly. Finally, we showed that IDAC is sensitive to modulation by comparing the resting state to the stimulus presentation.

\section{Materials and Methods}

\section{Study Population}

A total of 41 participants completed the imaging protocol (mean age of 36.2 years, SD 6.0 and range 32 years, 27 males). Individuals with relevant medical or neurologic symptoms, substance abuse or psychiatric illness and subjects undergoing medical treatment were not considered eligible for inclusion in the study. The study was conducted according to the principles expressed in the Declaration of Helsinki and was approved by the Clinical Research Ethical Committee of the Parc de Salut Mar of Barcelona. Written informed consent for fMRI assessment and subsequent analyses was obtained from every participant.

\section{MRI acquisition}

1.5 Tesla Signa Excite system (General Electric, Milwaukee, WI, USA) equipped with an eightchannel phased-array head coil and single-shot echo-planar imaging (EPI) software was used. The imaging protocol involved the following sequences:

Anatomical 3D. High-resolution anatomical images were obtained using an axial T1-weighted three-dimensional fast spoiled gradient inversion recovery-prepared sequence. A total of 134 
contiguous slices were acquired with repetition time $11.9 \mathrm{~ms}$; echo time $4.2 \mathrm{~ms}$; flip angle $15^{\circ}$; field of view $30 \mathrm{~cm}$; 256 x 256 pixel matrix; slice thickness $1.2 \mathrm{~mm}$.

Functional MRI. The functional MRI sequence consisted of gradient recalled acquisition in the steady state with repetition time $2000 \mathrm{~ms}$; echo time $50 \mathrm{~ms}$; pulse angle $90^{\circ}$; field of view $24 \mathrm{~cm} ; 64$ x 64-pixel matrix; slice thickness $4 \mathrm{~mm}$ (inter-slice gap, $1.5 \mathrm{~mm}$ ). Twenty-two interleaved slices were prescribed parallel to the anterior-posterior commissure line covering the whole-brain. Two 6min continuous scans with 180 whole-brain EPI volumes were acquired for each participant. The first four (additional) images in each run were discarded to allow magnetization to reach equilibrium. The first scan was acquired in the resting state for which the participants were instructed to relax, stay awake and lie still without moving, while keeping their eyes closed throughout. The second scan involved sustained visual-auditory stimuli presentation. The participants were shown a video composed of slides with happy faces and music showing a rapid tempo (Beethoven's $6^{\text {th }}$ Symphony "Pastoral") using MRI compatible goggles and headphones (VisuaStim Digital, Resonance Technology, USA).

\section{Image pre-processing}

Anatomical and functional images were all visually inspected before analysis by a trained operator to detect possible acquisition artifacts. Functional MRI images were slice-time corrected and realigned. The resulting affine realignment parameters were used for scrubbing, i.e. discarding motion-affected volumes (Power et al., 2014). For each subject, inter-frame motion measurements (Pujol et al., 2014a) served as an index of data quality to flag volumes of suspect quality across the run. At time points with inter-frame motion $>0.2 \mathrm{~mm}$, the corresponding volume, the immediately preceding and the succeeding two volumes were all discarded. Using this procedure, the average number of censored volumes per acquisition was 4\% (SD, 9.9\%). EPI volumes were then coregistered to their anatomical images with an affine transformation using Statistical Parametric 
Mapping (SPM). A warping matrix was also estimated for every subject to match a group template created from the 3D anatomical individual acquisitions and then to the Montreal Neurological Institute (MNI) space using DARTEL normalization (Ashburner, 2007).

Analyses were carried out on every subject's native space after EPI volumes were all realigned to their mean. EPI volumes were re-sliced to $3 \times 3 \times 3 \mathrm{~mm}$. Estimated DARTEL normalizations to the MNI space mentioned above were applied to the IDAC results (see below) to enable group inferences.

Analyses were conducted in a gray matter mask split into left and right hemispheres, so that no adjacent voxels from the medial regions of one hemisphere would be locally associated with those from the other hemisphere. The two hemispheres were brought back together once the IDAC values had been calculated. The left and right hemisphere gray matter masks were obtained by setting a threshold of $\mathrm{p}>0.4$ on the gray matter probability maps obtained from the DARTEL group template. As analyses were carried out in every subject's native space, the template masks were backtransformed with the inverse estimated normalization. We preferred applying an inverse normalization to the group-level gray matter masks as opposed to defining individual masks in the native gray matter segmentation to avoid important edge effects.

We repeated the analysis using a whole-brain mask that was not split into separate hemispheres and that included CSF and white matter tissue to assess the impact of the masking choice. The very few differences we found are reported in the supplementary material.

Analyses were also repeated with and without isotropic smoothing on the EPI volumes prior to the computation of IDAC values voxelwise. When applied, the smoothing involved convolving the image with a $4 \times 4 \times 4 m^{3}$ full width at half maximum (FWHM) Gaussian kernel. The results 
reported in the main text are obtained with unsmoothed EPIs, but results using smoothed EPIs are reported in the Supplementary Material.

Filtering and de-noising of data. All fMRI time series were band-passed with a Discrete Cosine Transform (DCT) filter letting through frequencies in the $0.01-0.1 \mathrm{~Hz}$ interval. We regressed all time series on the 6 rigid body realignment parameters and their 6 first-order derivatives, and on the average white matter and CSF signal extracted from the native tissue masks. To assess the robustness of our analyses to the selected de-noising strategy, we repeated them introducing changes in the nuisance design matrix. Small or moderate changes were found, not compromising the major clusters of our results (see Supplementary Material).

\section{Definition of Iso-Distant Average Correlation (IDAC)}

We defined the concept of "Iso-Distant Average Correlation" (IDAC) to describe the pattern of correlation decay in the close vicinity of a voxel. $I D A C_{i}(h)$ was consequently defined as the average temporal correlation of voxel $i$ with all the voxels located at a given distance lag $h$. fMRI data sets being a discrete sample, any distance lag $h$ must be necessarily transformed into a discrete isodistant interval $H_{k}=\left[h_{k}, h_{k}+1\right)$, with $h_{k}$ being a set of successively increasing distances covering the whole vicinity of a given voxel (see Figure 1).

The set of iso-distant intervals $H_{k}$ were selected so that temporal correlations were mainly positive, decreased monotonically and in which horizontal axon collaterals were considered likely to form local networks. For the present study, we chose the distances: $0,5,10,15, \ldots, 30 \mathrm{~mm}$. These 7 distances defined 7-1=6 iso-distant intervals, with constant thicknesses but increasing number of voxels (see Figure 1-Right). 
IDAC values could have been calculated for farther iso-distant intervals as well as within thinner intervals, providing a richer characterization of IDAC curves (but also noisier ones). However, beyond a 30mm-radius, negative correlations might also begin to be prevalent and give way to cancellation phenomena.

We first computed a correlation matrix $\boldsymbol{C}$ of Pearson coefficients comparing the BOLD time course of all the voxels in our study mask with each other's.

$$
C_{i, j}=\frac{\sum_{k=1}^{M}\left(Y_{i, k}-\bar{Y}_{l}\right) \cdot\left(Y_{j, k}-\bar{Y}_{\jmath}\right)}{\sqrt{\sum_{k=1}^{M}\left(Y_{i, k}-\bar{Y}_{l}\right)^{2}} \cdot \sqrt{\sum_{k=1}^{M}\left(Y_{j, k}-\bar{Y}_{\jmath}\right)^{2}}}
$$

where $M$ is the length of the BOLD time series and $i$ and $j$ index all the voxels entering our study mask. We then transformed the Pearson correlation matrix $\boldsymbol{C}$ into a gaussian distributed z-score correlation matrix $\boldsymbol{Z}$ by applying a Fisher transform.

$$
Z_{i, j}=\frac{\sqrt{M-3}}{2} \cdot \ln \left(\frac{1+C_{i, j}}{1-C_{i, j}}\right)
$$

We obtained then $I D A C_{i}\left(h_{k}\right)$ by averaging the correlation coefficients of voxel $i$ with all the voxels $j$ belonging to the interval $H_{k}$.

$$
\underline{I D A C_{i}}\left(h_{k}\right)=\frac{\sum_{j \epsilon H_{k, i}} Z_{i, j}}{N_{k, i}}
$$

In short, IDAC values are defined as the mean correlation z-score between one voxel's fMRI signal and the fMRI signal of all the voxels within the iso-distant interval $H_{k, i}$. Note that, for a given 
distance lag $k$, the number of voxels within the concentric iso-distant interval $N_{k, i}$ is not necessarily the same for every voxel $i$ due to the edge effects of the study mask.

Of course, as the distance lag $h_{k}$ increases, $I D A C_{i}\left(h_{k}\right)$ will drop, defining a decreasing curve in average local correlations. Figure 1 (left) shows the spatial distribution of the correlation z-scores within a range of $30 \mathrm{~mm}$ to the origin coordinate ("seed") for a subject in its native space. As we can see, the correlation decays almost monotonically as the distance lag is increased.

\section{Descriptive and statistical interpretation of IDAC}

IDAC curves can largely benefit from a representation where more than one distance lag is plotted or mapped at the same time.

Group-averaged IDAC Plots. As is usually done with correlograms, we plotted the correlation coefficient on the ordinate and the spatial lag on the abscissa. Group-averaged IDAC plots were straightforwardly generated by averaging IDAC values across subjects voxelwise. The group variance of every IDAC value was also used to shade an interval confidence for the group-averaged values. Comparing group-averaged IDAC plots from different brain areas allowed us to visually inspect those distances at which local functional connectivity varies the most between two anatomical regions (Figure 4). Likewise, the plot of different IDAC curves together also helped us visualize the differences in functional connectivity that one same brain area present during different functional states (applied in Figure 6 and 7).

Group-averaged Multi-distance IDAC maps. It is also possible to generate multi-distance IDAC maps obtained from the overlay of IDAC values from three different distance intervals using an RGB color codification. RGB color channels permit the display of three IDAC values obtained from different distance lags at the same time. In all the figures in our study (Figures 3, 4, 6 and 7), 
we chose RED for interval 5-10mm, GREEN for 15-20mm and BLUE for 25-30mm. The rationale behind mixing colors is that overlapping primary colors will produce secondary and tertiary colors, each of which characteristic of a different IDAC curve shape. It is true that RGB color coding grossly simplifies the information in IDAC curves down to a three-dimensional vector but it offers the insight of capturing the anatomical distribution of the measure.

To establish a color-coding scale that was not exceedingly sensitive to outliers, we used for every RGB color channel a maximum color saturation for values above the 90th percentile and a minimum color saturation (i.e. black) for values below the 10th percentile. Percentiles were calculated independently for every distance lag and considering the distribution of the groupaveraged IDAC values over all the voxels entering the study mask.

We produced a group-averaged multi-distance IDAC map from the resting-state acquisition of 41 control subjects and displayed it on 3D and surface renderings (see Figures 2, 3 and 4).

\section{Multivariate tests for comparing group-level IDAC curves.}

The comparison of our IDAC curves was conducted with the natural extension of univariate Student's t-tests, which are multivariate Hotelling's $\mathrm{T}^{2}$ tests. Our IDAC curves were parametrized by six values obtained from six iso-distant intervals, so that $\operatorname{IDAC}=\left(\operatorname{IDAC}\left(\mathrm{h}_{1}\right), \operatorname{IDAC}\left(\mathrm{h}_{2}\right), \ldots\right.$, IDAC $\left(\mathrm{h}_{6}\right)$ ) was a random vector, that was assumed to satisfy all the conditions for a Hotelling $\mathrm{T}^{2}$ test (Anderson, 1986). These conditions are exactly the same assumptions as the ones required in a univariate Student's t-test with the sole difference that here the random variable is not a scalar, but a vector sampled from a multivariate gaussian population. 
We used a paired Hotelling's $T^{2}$ test to compare the audio-visual and the rest condition voxelwise (Figure 6 and 7). Additionally, a paired Hotelling's $T^{2}$ test was also used to compare IDAC curves obtained from adjacent brain regions (Figure 4).

IDAC Hotelling's $\boldsymbol{T}^{2}$ Maps. When we compared between the audio-visual and rest condition, paired Hotelling's $T^{2}$ tests were conducted voxelwise, and therefore could be represented as statistical brain maps (see Figure 5).

Hotelling's $T^{2}$ maps, like other statistical maps, require a correction for multiple comparisons given that every voxel is a different statistical test strongly dependent on the tests of the neighboring voxels. We addressed this issue with a Montecarlo simulation of the cluster size distribution of significant voxels conducted with the AFNI 3dClustSim function. Our Montecarlo simulation was based on the assumption that the final Hotelling's $T^{2}$ statistical map had a spatial autocorrelation function (ACF) given by a Gaussian function.

The simulation parameters used in our Montecarlo simulation were: 45,653 voxels in the simulation mask, cluster forming probability threshold of $\mathrm{p}<0.005$, with estimated FWHM of 9.0x9.7x9.4mm ${ }^{3}$. The above procedure resulted in a threshold cluster size of 52 voxels $\left(1.4 \mathrm{~cm}^{3}\right)$, corresponding to a corrected significance level of 0.05 . Such results are valid for the Hotelling's $T^{2}$ map of Figure 5, but each sensitivity analyses repeated in the Supplementary Material required its own simulation whose details are reported there.

\section{Multi-distance paired t-student maps}

Hotelling's $T^{2}$ statistical maps can provide us with a tool to detect areas where local connectivity is significantly different under different conditions, but unfortunately they can only test an omnibus hypothesis and cannot reveal us the precise distance lags or the sign where these differences occur. 
A way to address this is to generate repeated Student's t-maps using IDAC values from different distance lags and display them together using again an RGB color-code.

After we had produced the paired Hotelling's $T^{2}$ map, we generated three different paired t-test maps comparing IDAC values from intervals 5-10mm, 15-20mm and 25-30mm, and overlaid their results on different RGB color channels (see Figure 6 and 7). Considering this procedure as a posthoc explanatory analysis, we masked the multi-distance t-test maps with the significant clusters from the Hotelling's $T^{2}$ map results. The RGB color-coding convention was chosen the same as for group-averaged multi-distance IDAC maps.

\section{Paired t-student profile plots}

Multi-distance t-student maps are necessarily limited to three distance lags, three being the number of RGB colors that we can meaningfully mix up. However, we can further generate paired t-student profile plots for particular regions or voxels. In this case, we are not limited to only three distances, but we can extent the calculation to all the distance lags of our IDAC curves. T-student profile plots can help us determine the distance lags at which local similarity differences occur as well as the sign of these differences, in an even more detailed way than multi-distance t-student maps can, despite the clear disadvantage that here we lose all the precious anatomical information we had in multi-distance t-maps. In this study, we took advantage of paired t-student profile plots to compare between different anatomical regions during one same condition (Figure 4) as well as to compare between different conditions within one same anatomical region (Figure 6 and 7). We generally plotted these paired t-test profile plots on the same x-axis as the two group-averaged IDAC curves being compared (see Figures 4, 6 and 7).

\section{Results}

\section{Group-average IDAC multi-distance maps}


IDAC values neatly discriminated between gray and white matter tissue. Unsurprisingly, gray matter IDAC showed much higher values at all distance lags than those extracted in white matter regions. In addition to that, IDAC values were able to reveal further anatomical distinctions within the gray matter tissue, provided that IDAC values from different distance lags were considered together.

The overlay of three IDAC values belonging to increasingly distant lags using RGB color coding gave place to an eloquent cortical segmentation of the brain into gross anatomo-functional brain regions. Figure 2 shows the group-averaged IDAC maps generated from three different distance lags $(5-10 \mathrm{~mm}, 15-20 \mathrm{~mm}$ and $25-30 \mathrm{~mm})$ displayed separately and then overlaid together (multidistance). Figure 3 renders the same group-average multi-distance IDAC map on a cortical surface.

\section{Statistical comparison of cortical locations according to their IDAC}

Using the exploratory multi-distance IDAC map of Figure 2 and 3, we selected six pairs of ROIs that were adjacent to one another (all ROIs compared were less than $2 \mathrm{~cm}$ apart), that belonged to distinct regions (Talairach and Tournoux, 1988), and that multi-distance color mapping was able to differentiate. Then, we compared the selected pairs using a paired Hotelling's T2 test. Figure 4 illustrates the ROI's locations and the statistical significance of the differences.

Additionally, in three out of the six comparisons in Figure 4, we generated group-average IDAC plots and paired t-Student profile plots to determine which distances were mainly responsible for the differences. For example, sensorimotor and premotor ROIs turned out to vary mostly within the $5-20 \mathrm{~mm}$ proximity range; the posterior and the anterior insula within the $10-25 \mathrm{~mm}$ range; whilst the anterior cingulate cortex and the paracingulate cortex at more peripheral local ranges, $25-30 \mathrm{~mm}$.

\section{Comparison of different brain functional states}


We also compared IDAC, voxelwise, from a continuous eyes-closed resting state with a continuous audio-visual presentation. Figure 5 shows the paired Hotelling's $\mathrm{T}^{2}$ statistical map testing potential differences in IDAC values between the two conditions. Significant differences in IDAC curves mainly involved bilateral areas and large clusters of the visual cortex, auditory cortex, the anterior insula, posterior areas of the default mode network and lateral frontal areas (Table 1).

Like an $\mathrm{F}$ statistic in the context of an ANOVA, significant Hotelling's $\mathrm{T}^{2}$ clusters cannot distinguish between the sign of differences. Furthermore, it cannot inform us either of the range of distances responsible for the differences. Differences could arise from higher values in certain distance lags and lower values in other distance lags (if plotted, IDAC curves would, in such cases, cross in a conspicuous way).

To address this question, we generated multi-distance one tailed t-student maps whose results were restricted (masked) within significant clusters from the Hotelling's $\mathrm{T}^{2}$ analyses. Red, green and blue color channels were used for $5-10 \mathrm{~mm}, 15-20 \mathrm{~mm}$ and $25-30 \mathrm{~mm}$ distance lags respectively (see METHODS). Figure 6 shows that IDAC values during the visual-auditory stimulation significantly increased in the visual cortex, the fusiform gyrus and the auditory cortex. By contrast, they decreased in the posterior cingulate cortex, anterior insula, opercular region and the lateral-ventral aspect of the frontal cortex (Figure 7).

Such results strongly suggest that IDAC values are indeed modulated by evoked brain activity. The maps largely coincide, although they are clearly not identical, with traditional activation/deactivation maps in face presentation tasks (Fusar-Poli et al., 2009; Pujol et al., 2009). However, quite unexpectedly, some frontal areas which had been reported in the literature to activate during a face visual task, were here recorded as IDAC decreases (Figure 7). Such frontal 
decreases in local functional connectivity particularly involve medium and far distance lags (1530mm).

The multi-distance mapping technique using RGB color coding also allowed us to spot which local distances were responsible for the differences. Figure 6 shows that increases in the innermost range of functional connectivity (colors red and yellow) involved areas closer to the primary visual cortex, together with the center of the auditory cortex. At further distance ranges (coded in white, cyan and blue), increases in IDAC values covered extrastriate visual areas and the fusiform gyrus. Arguably, the fact that the most associative areas in the visual and auditory cortex are mainly modulated in the long ranges of local connectivity also seems biologically plausible.

\section{Discussion}

In an effort to draw attention to the biological significance of the spatial structure of local activity similarities in fMRI data, we proposed a multivariate measure, called IDAC, that derives from the popular concept of spatial correlogram. IDAC computation voxelwise is easy to reproduce by other research teams, computationally little intensive, and easy to visualize either in plots or displayed as multi-distance maps.

An IDAC curve is generated by the average fMRI temporal correlation of one given voxel with its surrounding voxels at different spatial lags. Given that fMRI data sets are not continuous, IDAC curves must be generated by sampling a vector whose length depends on the number of iso-distant intervals defined. We hypothesized that different brain areas would have characteristic IDAC curves and that these curves would be functionally modulated whenever the region's local connectivity changed. 
IDAC curves can be compared statistically, voxelwise or region to region, using multivariate statistics. In this study, we used a paired Hotelling's $\mathrm{T}^{2}$ test to compare different values from IDAC curves jointly between a continuous stimulation and a resting state and, also, to compare IDAC values between two same regions during the same condition. In general, for single contrast tests, the natural test statistic would still be a Hotelling's $\mathrm{T}^{2}$, whereas the multivariate analogue of an Fstatistic would be a Wilks's $\Lambda$ (Worsley et al., 2004). Once multivariate statistical mapping has established those brain areas that show significant changes in local functional connectivity, post-hoc multi-distance IDAC mapping, though limited to the display of only three distance lags, is a display strategy that is able to provide anatomically rich representations of the dynamics of local functional connectivity at different local scales.

Our first important result on applying IDAC to a testing set of 41 acquisitions was that IDAC values belonging to different distance lags, when displayed together in multi-distance maps using RGB color coding, were able to parcel the brain cortex into regions arguably resembling those we can find in traditional anatomo-functional brain atlases. For example, the visual cortex is distinguished from the temporal and parietal lobes, and perirolandic cortex from premotor and prefrontal areas (Figure 3). In the occipital lobe, the dominant pattern involves high local connectivity at all local distance ranges (typically white in the maps), whereas the angular (Brodmann area, BA39) and supramarginal gyri (BA40) of the inferior parietal lobule (Talairach and Tournoux, 1988) are both mostly connected at short and medium local distances (yellow). See Supplementary Figure 5 for more detail. Remarkably, the yellow pattern was also found in the caudal aspect of the posterior cingulate cortex. In the typical long-distance (brain-scale) functional connectivity mapping, although both the core posterior cingulate cortex and the angular gyrus are strongly coupled in the context of the default mode network, the network does not include the supramarginal gyrus (Harrison et al., 2008). 
Therefore, IDAC mapping has not a complete match with a single anatomical, histological or functional brain parcellation, but the correspondence is partial and mixed. This notion is also illustrated in the primary motor area and primary somatosensory area that appear to be relatively highly connected at all local distances (Supplementary Figure 5), although both areas show very distinct cytoarchitectonic features (Garey, 2006). However, the motor cortex and the somatosensory cortex have notable similarities in terms of myeloarchitecture, with high cortical myelin content (Glasser et al., 2014).

Of note, by estimating differences between local and distant degree connectivity, Sepulcre et al. (2010) also obtained a degree of brain parcellation into major anatomo-functional regions. Interestingly, as in our maps, the motor cortex was jointly identified with the somatosensory cortex and the angular gyri with the supramarginal gyri.

Our second important result was that local functional connectivity described with IDAC is not a static characteristic of the brain tissue. We showed that IDAC curves can be critically modulated in relevant areas with changes in experimentally controlled brain states. When we compared an eyesclosed resting state versus a visual-auditory continuous stimulation, we discovered that a number of well-known activation-related areas (Fusar-Poli et al., 2009) showed significant increases in local correlations. At the innermost distance lags, we found increases in core visual areas, parts of the fusiform and the center of the auditory cortex; at more peripheral local regions, higher-level visual areas also came into play (fusiform gyrus and extrastriate areas). Such differential modulation of local functional connectivity between low- and high-level primary areas seems to us biologically convincing and suggests that the technique, by introducing a smooth spatial description of the integration phenomenon, introduces new possibilities of understanding that are not available in previous measures of local similarity. 
We also found important decreases during the audio-visual stimulation in areas known to deactivate during attention-demanding tasks, mainly the posterior cingulate cortex as part of the default mode network $(\mathrm{DMN})$. We found that this is particularly conspicuous at the shortest ranges $(\mathrm{R}<20 \mathrm{~mm})$ of local functional connectivity. A less predictable result was to find other areas, not directly associated with the traditional DMN anatomy, which also showed decreased local connectivity during stimulation. These areas mainly involved parts of the lateral frontal cortex, anterior insula and opercula bilaterally. Here, IDAC values were significantly lower at medium and long ranges (15-30mm). This finding is particularly challenging to our interpretation because some of these areas have been reported to "activate" in visual bloc task experiments (Fusar-Poli et al., 2009). Hence, our study shows that it is possible for a cortical area that is known to activate also to decrease its local functional connectivity at certain critical distances. This should not come as a surprise, since already in the seminal paper by Zang et al. (2004) where ReHo, perhaps the most popular measure of local similarity today, was first proposed, it was already found that local similarities increased ipsi-laterally during a finger-tapping task, instead of contra-laterally as was expected from traditional univariate activation analyses. Thus, IDAC mapping cannot merely be taken as a surrogate for activation experiments.

Local similarity analyses are well-suited to studying psychological and neurological conditions such as sustained emotional states, moods, hormone or drug level effects that are difficult to test with activation/deactivation analyses, because of the necessity for the experimenter to force the participant to change from one condition to another at a fast pace $(<30 \mathrm{~s})$.

The underlying biological phenomena responsible for changes in local activity similarities remain partially unknown. A number of studies have pointed out that the strength of local correlations may be associated with average regional metabolic activity, as can be accessed indirectly with arterial spin labelling (ASL) MRI (Liang et al., 2013) or, more directly, with PET (Aiello et al., 2015, 
Nugent et al., 2015). In particular, Liang et al. (2013) found a strong coupling between blood supply and brain functional topology, which, furthermore, could be modulated in response to task demands. Others have preferred to interpret it from the connectivity paradigm (Rubinov and Sporns, 2010; Sporns, 2013; Tomasi et al., 2010). Both assumptions may be appropriate and local fMRI signal integration may have to do with both local connectivity and local coactivation. Indeed, we know that at a microscopic level, a spatially broad intra-columnar and cortico-cortical synapsing cascade is responsible for amplification, modulation and processing of a single input signaling.

We reported results obtained with the least possible processing sophistication to make IDAC mapping an appealing technique to other laboratories. A more sophisticated processing pipeline may perhaps be pertinent in future developments. Some important processing variations that were likely to affect IDAC values, such as image smoothing, use of gray matter masking, proportional scaling of IDAC values at a subject level, etc. were also appraised. These results and the few differences they generated are reported in the Supplementary Material.

Being also concerned by the robustness of IDAC maps to differences in acquisition resolution, we also calculated group-averaged IDAC maps on high resolution fMRI data $\left(2 \times 2 \times 2\right.$ and $\left.3 \times 3 \times 3 m^{3}\right)$. We used the resting-state data of a subsample of the publicly available NKI-Rockland sample, after matching it by sample size, age and sex to our study sample. Almost imperceptible differences were found between multi-distance IDAC maps from the two resolutions and relatively few differences between those from our sample and the validation sample. The results are amply described in the Supplementary Material.

A processing variation that could be explored in the future is the effect of using more realistic nonEuclidian "cortical" iso-distant intervals. This could be achieved, for instance, by defining geodesic distances over the cortex surface or using a closest path search algorithm confined to every 
participant's native gray matter mask. However, a word of caution is necessary. The use of nonEuclidian metrics raises additional problems: non-Euclidian distances might indeed be more suitable to model the spatial autocorrelation of data arising from neural origin but would unfairly treat the spatial autocorrelation due to smoothed noise (the main contributor to IDAC measures), which is assumed to be relatively isotropic and not related to cortical distances.

Another concept worth mentioning is that IDAC does not account for the eccentricity of correlations with neighboring voxels along the different orientation axes. IDAC computation requires the definition of iso-distant intervals that do not take into account the fact that correlations in these intervals will not in general have the same strength along different reference axes. In other words, the 3D gradient of local correlations to a center point (a seed) is anisotropic. Taking this anisotropy into account could open up a new and interesting field of analysis.

\section{Conclusion}

We showed that the smooth spatial structure of local fMRI signal correlations as it can be described with IDAC is a promising source of biologically relevant information. IDAC curves can be viewed as the signature of a given brain location under a certain brain condition. IDAC curves can be compared and studied, even voxelwise, using the natural generalizations of univariate parametric statistics to their multivariate homologues as we did by replacing a t-student by a Hotelling's $\mathrm{T}^{2}$. Furthermore, post-hoc multi-distance IDAC mapping, although it can only test and display results combining three distance lags at a time, are rich representations of the dynamics of local functional connectivity the form of statistical maps. 


\section{Acknowledgements}

This work was supported in part by the Carlos III Health Institute grant PI10/02206 and the I+D+I grant PSI2014-53524-P. We thank the Agency of University and Research Funding Management of the Catalonia Government for their participation in the context of Research Group SGR SGR20171198.

\section{Author Disclosure Statement}

No competing financial interests exist. 


\section{References}

Abeles M. 1982. Local Cortical Circuits. Berlin: Springer.

Aiello M, Salvatore E, Cachia A, Pappatà S, Cavaliere C, Prinster A et al. 2015. Relationship between simultaneously acquired resting-state regional cerebral glucose metabolism and functional MRI: a PET/MR hybrid scanner study. Neuroimage 113:111-121.

Amir Y, Harel M \& Malach R. 1993. Cortical hierarchy reflected in the organization of intrinsic connections in macaque monkey visual cortex. J Comp Neurol 334:19-46.

Anderson T W. 2003. An Introduction to Multivariate Statistical Analysis, 3th edition. New York: Wiley.

Ashburner J. 2007. A fast diffeomorphic image registration algorithm. Neuroimage 38:95-113.

Baumgartner R, Somorjai R, Summers R, Richter W. 1999. Assessment of cluster homogeneity in fMRI data using Kendall's coefficient of concordance. Magn Reson Imaging 17:1525-1532

Bernier M, Croteau E, Castellano CA, Cunnane SC, Whittingstall K. 2017. Spatial distribution of resting-state BOLD regional homogeneity as a predictor of brain glucose uptake: A study in healthy aging. Neuroimage 150:14-22.

Burkhalter A. 2016. The Network for Intracortical Communication in Mouse Visual Cortex. In: 
Kennedy H, Van Essen DC, Christen Y (eds.) Micro-, Meso- and Macro-Connectomics of the Brain. Cham $(\mathrm{CH})$ : Springer.

Felleman DJ \& Van Essen DC. 2011. Distributed hierarchical processing in the primate cerebral cortex. J Neurophysiol 106:1125-1165.

Fusar-Poli, P Placentino A, Carletti F, Landi P, Allen P, Surguladze S, Benedetti F, et al. 2009. Functional atlas of emotional faces processing: a voxel-based meta- analysis of 105 functional magnetic resonance imaging studies. J Psychiatry Neurosci 34:418-432.

Garey LJ. 2006. Brodmann's Localisation in the Cerebral Cortex. New York: Springer Science.

Glasser, MF, Goyal, MS, Preuss, TM, Raichle, ME, Van Essen DC. 2014. Trends and properties of human cerebral cortex: correlations with cortical myelin content. Neuroimage. 93:165-715.

Grinvald A, Omer D, Naaman S, Sharon D. 2015. Imaging the Dynamics of Mammalian, Neocortical Population Activity In-Vivo. Adv Exp Med Biol 859:243-271.

Harrison BJ, Pujol J, López-Solà M, Hernández-Ribas R, Deus J, Ortiz H, et al. 2008. Consistency and functional specialization in the default mode brain network. Proc Natl Acad Sci USA 105:97819786.

Kendall, M., Gibbons, J.D. 1990. Rank Correlation Methods. 5th Editioin. London: Oxford Univ Press. 
Liang J, Xu W, Geng X \& Wu JY. 2015. Monitoring population membrane potential signals from neocortex. Adv Exp Med Biol 859:171-196.

Liang X, Zou Q, He Y, Yang Y. 2013. Coupling of functional connectivity and regional cerebral blood flow reveals a physiological basis for network hubs of the human brain. Proc Natl Acad Sci USA 110:1929-1934.

Liu Y et al. 2008. Regional homogeneity, functional connectivity and imaging markers of Alzheimer's disease: a review of resting-state fMRI studies. Neuropsychologia 46:1648-56.

Liu D, Yan C, Ren J, Yao L, Kiviniemi VJ, Zang Y. 2010. Using coherence to measure regional homogeneity of resting-state FMRI signal. Front Syst Neurosci 4:24.

Nugent AC, Martinez A, D'Alfonso A, Zarate A \& Theodore WH. 2015. The relationship between glucose metabolism, resting-state fMRI BOLD signal, and GABAA-binding potential: a preliminary study in healthy subjects and those with temporal lobe epilepsy. J Cereb Blood Flow Metab 35:583-591.

Power JD, Mitra A, Laumann TO, Snyder AZ, Schlaggar BL, Petersen SE. 2014. Methods to detect, characterize, and remove motion artifact in resting state fMRI. Neuroimage 84:320-341.

Pujol J, Harrison BJ, Ortiz H, Deus J, Soriano-Mas C, López-Solà M, et al. 2009. Influence of the fusiform gyrus on amygdala response to emotional faces in the non-clinical range of social anxiety. Psychol Med 39:1177-1187. 
Pujol J, Macià D, Blanco-Hinojo L, Martínez-Vilavella G, Sunyer J, de la Torre R, Caixàs A et al. 2014a. Does motion-related brain functional connectivity reflect both artifacts and genuine neural activity? Neuroimage 101:87-95.

Pujol J, Macià D, Garcia-Fontanals A, Blanco-Hinojo L, López-Solà M, Garcia-Blanco S, et al. 2014b. The contribution of sensory system functional connectivity reduction to clinical pain in fibromyalgia. Pain 155:1492-1503.

Pujol J, del Hoyo L, Blanco-Hinojo L, de Sola S, Macià D, Martínez-Vilavella G, Amor M, et al. 2015. Anomalous brain functional connectivity contributing to poor adaptive behavior in Down syndrome. Cortex 64:148-56.

Rubinov M, Sporns O. 2010. Complex network measures of brain connectivity: uses and interpretations. Neuroimage 52:1059-1069.

Sepulcre J, Liu H, Talukdar T, Martincorena I, Yeo BT, Buckner RL. 2010. The organization of local and distant functional connectivity in the human brain. PLoS Comput Biol 6(6):e1000808.

Sporns O. 2013. Network attributes for segregation and integration in the human brain. Curr Opin Neurobiol 23:162-171.

Talairach J, Tournoux P. 1988. Co-planar Stereotaxic Atlas of the Human Brain. New York, NY: Thieme Medical Publishers.

Tomasi D, Volkow ND. 2010. Functional connectivity density mapping. Proc Natl Acad Sci USA 107:9885-9890. 
Tomasi D, Volkow ND. 2011. Functional connectivity hubs in the human brain. Neuroimage 57:908-917.

Tomasi D, Volkow ND. 2012a. Resting functional connectivity of language networks: characterization and reproducibility. Mol Psychiatry 17(8):841-854.

Tomasi D, Volkow ND. 2012b. Aging and functional brain networks. Mol Psychiatry 17(5):471, 549-558.

Tomasi D, Volkow ND. 2012c. Abnormal functional connectivity in children with attentiondeficit/hyperactivity disorder. Biol Psychiatry 71:443-450.

Tomasi D, Wang R, Wang GJ, Volkow ND. 2014. Functional connectivity and brain activation: a synergistic approach. Cereb Cortex 24:2619-2629.

Tononi G, Sporns O, Edelman GM. 1994. A measure for brain complexity: Relating functional segregation and integration in the nervous system. Proc Natl Acad Sci USA 24:5033-5037.

Varela F, Lachaux JP, Rodriguez E, Martinerie J. 2001. The brainweb: phase synchronization and large-scale integration. Nat Rev Neurosci 4:229-239.

Worsley KJ, Taylor JE, Tomaiuolo F, Lerch J. 2004. Unified univariate and multivariate random field theory. Neuroimage 23(SUPPL. 1):189-195. 
Xu C, Li C, Wu H, Wu Y, Hu S, Zhu Y, et al. 2015. Gender differences in cerebral regional homogeneity of adult healthy volunteers: a resting-state FMRI study. Biomed Res Int. 2015:183074.

Zang Y, Jiang T, Lu Y, He Y, Tian L. 2004. Regional homogeneity approach to fMRI data analysis. Neuroimage 22:394-400. 


\section{Figures}

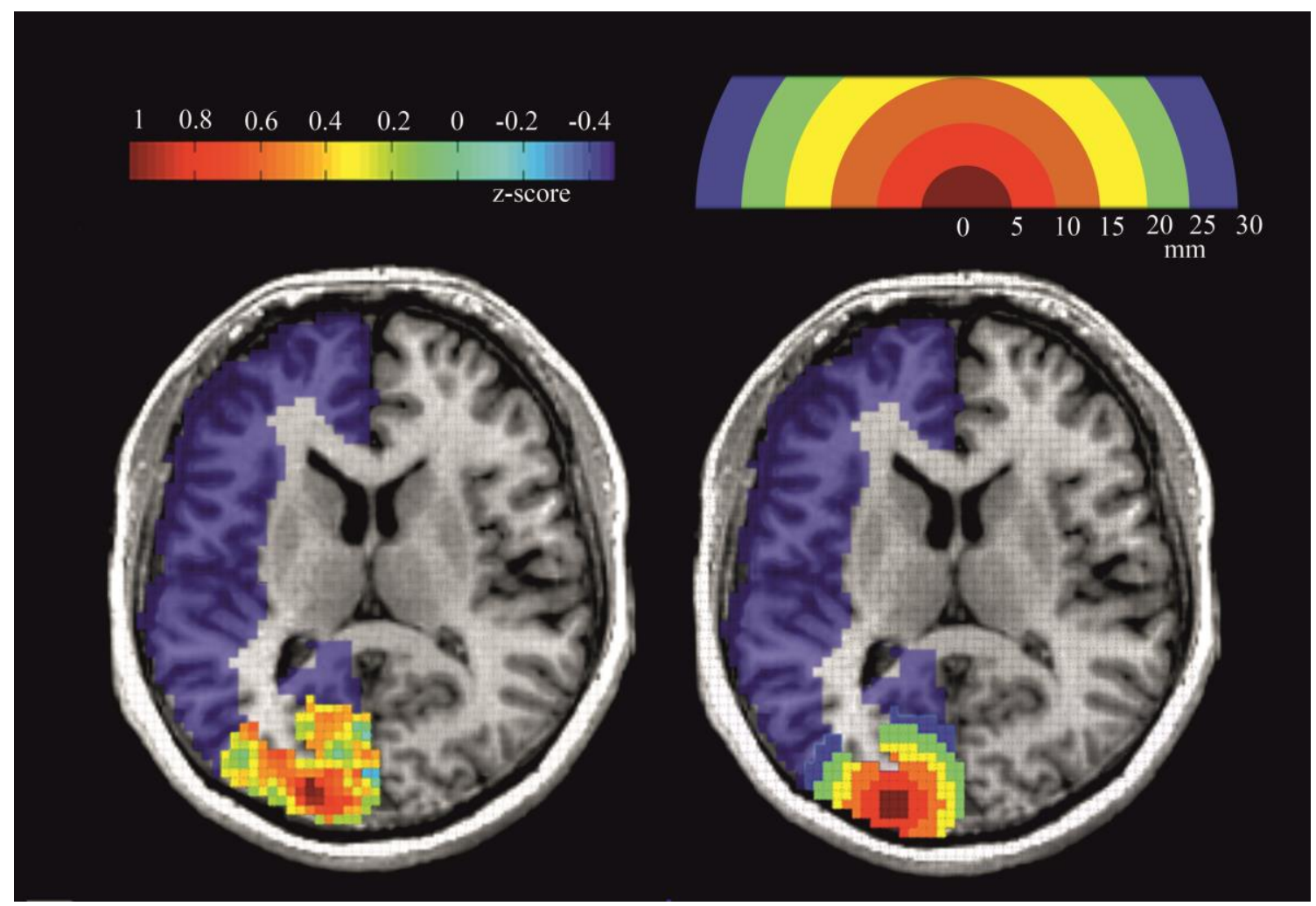

Figure 1. fMRI Temporal correlations between one voxel ("seed") and its neighboring peripheries present a characteristic decreasing spatial gradient.

LEFT: Fisher-transformed z-scores of a correlation map with a "seed" voxel in the visual area from a single subject. Voxel resolution is $3 \times 3 \times 3 \mathrm{~mm}$ and results are constrained to distance lags $\mathrm{h}_{\mathrm{k}}<30 \mathrm{~mm}$ and within the subject's native gray-matter mask (blue shade). RIGHT: Six different Iso-distant intervals as they are used to calculate different IDAC values in our study. 


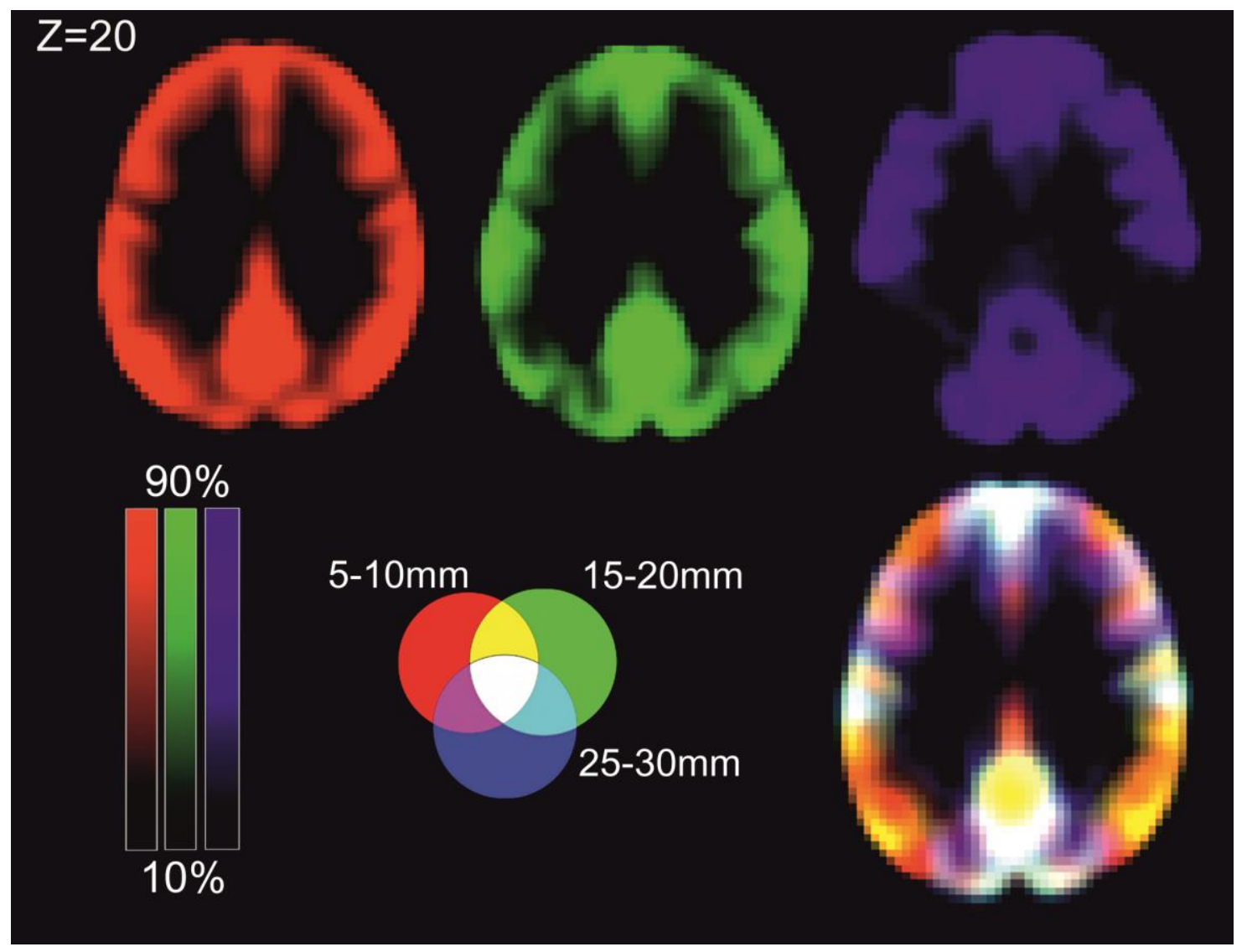

Figure 2. Formation of a group-averaged multi-distance IDAC map.

ABOVE: single color IDAC maps obtained from three different iso-distant intervals: RED for distances 5-10mm, GREEN for 15-20mm, BLUE for 25-30mm. IDAC values are calculated voxelwise as the group average of 41 acquisitions during a resting state. Color bar ranges are set using the histogram of the group-averaged IDAC values over all the voxels entering the study mask independently for each distance lag. BELLOW-LEFT: percentile color bar limits and diagram of RGB mixtures. BELLOW-RIGHT: result of overlapping the single-colored three IDAC maps from the top row. Note that all IDAC maps shown in this figure were generated without gray-matter masking to show that white matter and CSF tissue present much lower IDAC values at all distance lags than gray matter and therefore are shown in dark $(<10 \%)$. 


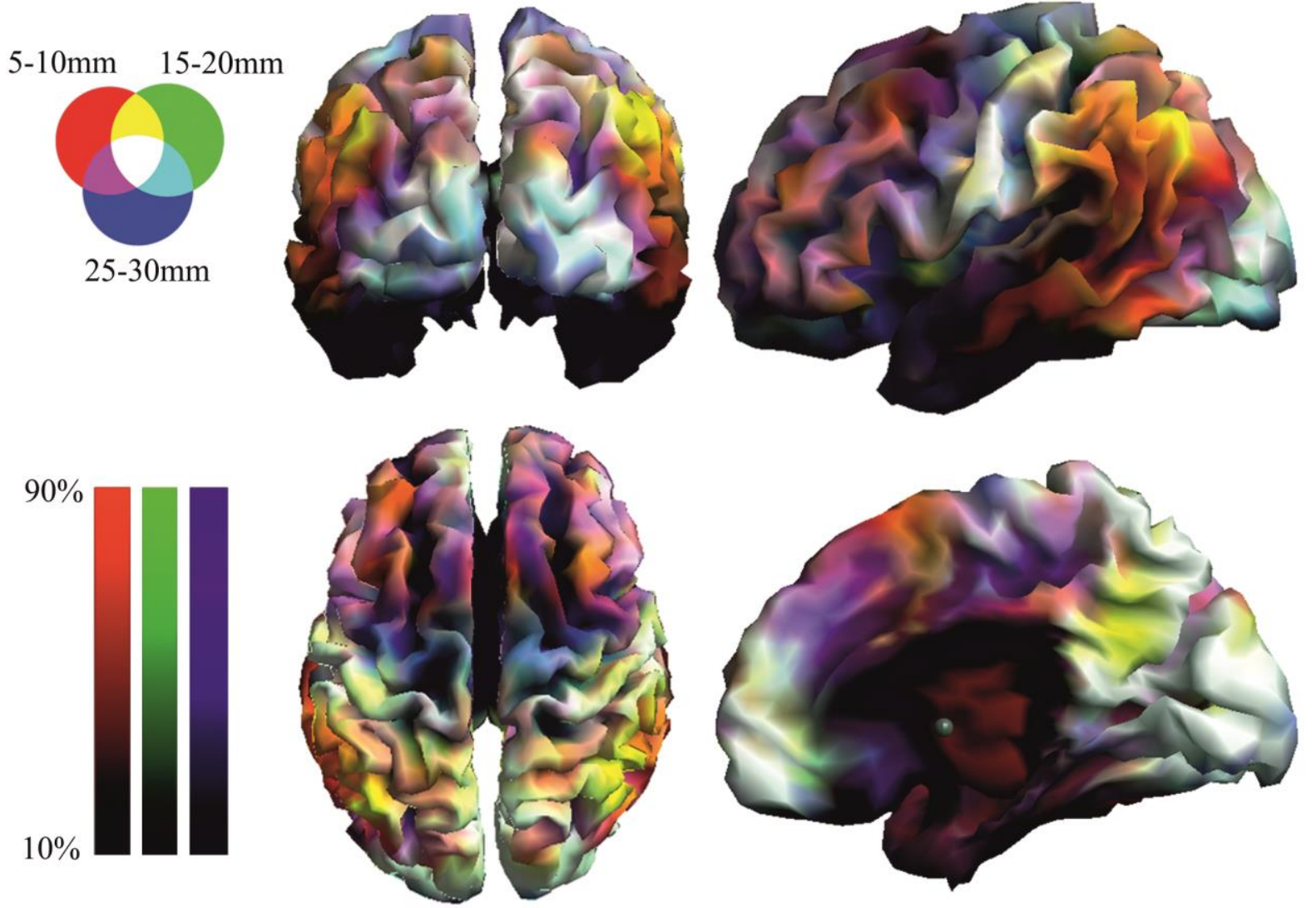

Figure 3. Group-averaged multi-distance IDAC map projected onto a cortical surface. RED for $\mathrm{r}=5-10 \mathrm{~mm}$, GREEN for $\mathrm{r}=15-20 \mathrm{~mm}$, BLUE for $\mathrm{r}=25-30 \mathrm{~mm}$. The color bar range is set using the histogram of the group-averaged IDAC values over all the voxels entering the study mask independently for each distance lag as in Figure 2. 


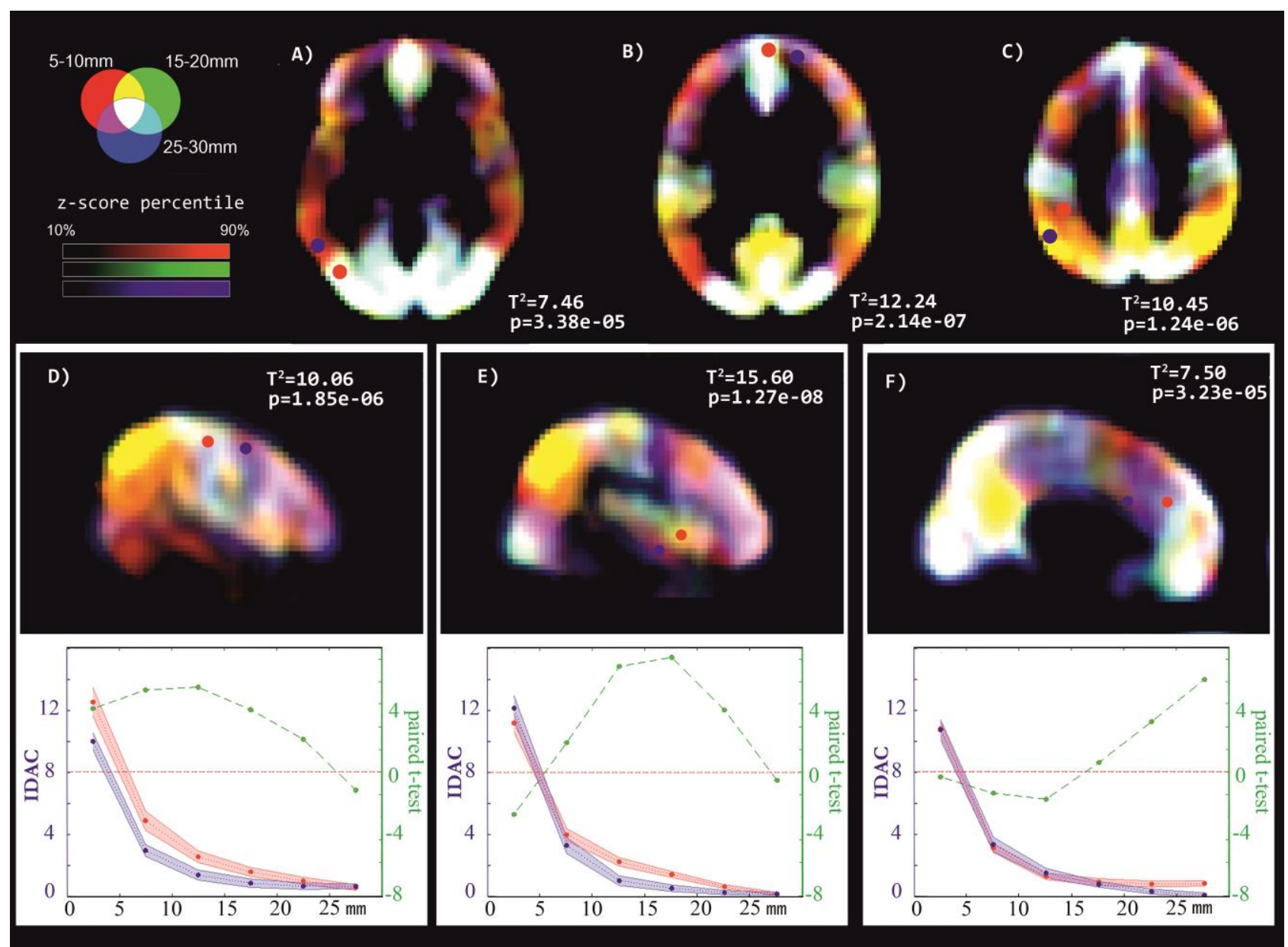

Figure 4. Comparison of IDAC curves from adjacent anatomical regions. Group-average multidistance IDAC maps with pairs of ROIs indicated with red and blue dots. Color bar ranges are set using IDAC percentiles (see Figures 2 and 3). A), B) and C): adjacent locations compared with paired Hotelling's $\mathrm{T}^{2}$ tests. D), E) and F): further adjacent locations compared with multivariate statistics complemented by group-averaged IDAC curves and t-student profile plots. Red IDAC curves are extracted from red dot ROIs and blue curves from blue dot ROIs. The shaded area in the plots represent the group mean $95 \%$ confidence interval for every group-averaged IDAC value calculated for $\mathrm{N}=41$ subjects. Dashed green lines are paired t-student profile plots comparing the two IDAC curves at every distance lag. Note that for $\mathrm{N}=41$ subjects, $\mathrm{t}>2.7$ corresponds to a $\mathrm{p}<0.005$. 


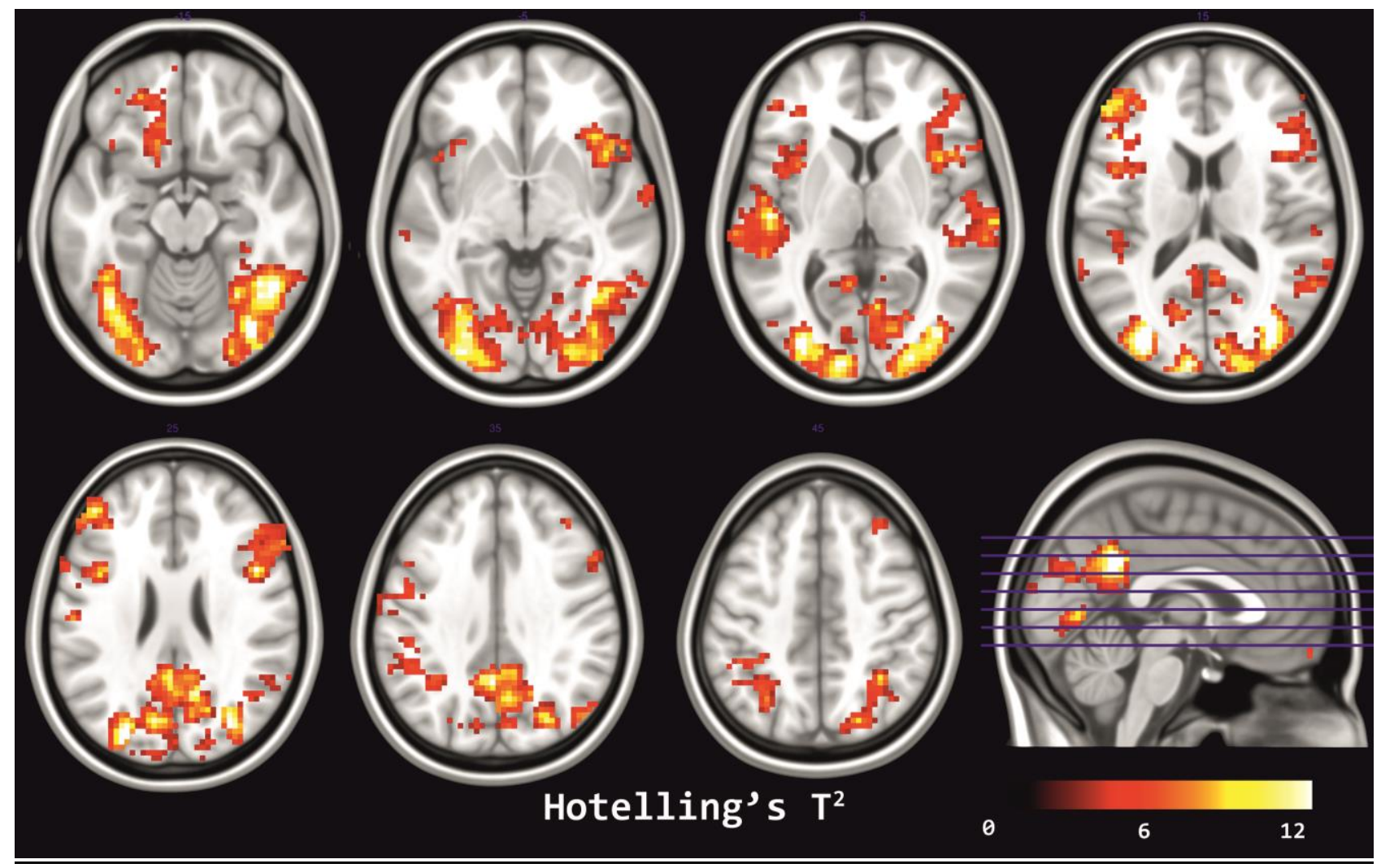

Figure 5. Omnibus comparison of IDAC values, audio-visual vs. rest

Paired Hotelling's $\mathrm{T}^{2}$ map comparing the audio-visual stimulation with the rest condition. Results are thresholded at cluster-size corrected significance level of 0.05 and include voxels where IDAC curves have significantly different shapes between the two conditions. Such differences may include both increases and decreases and may involve different distance lags of connectivity for one same voxel. Differences mainly involve the visual and auditory cortices, posterior areas of the default mode network, operculum, anterior insulae and frontal cortex. 

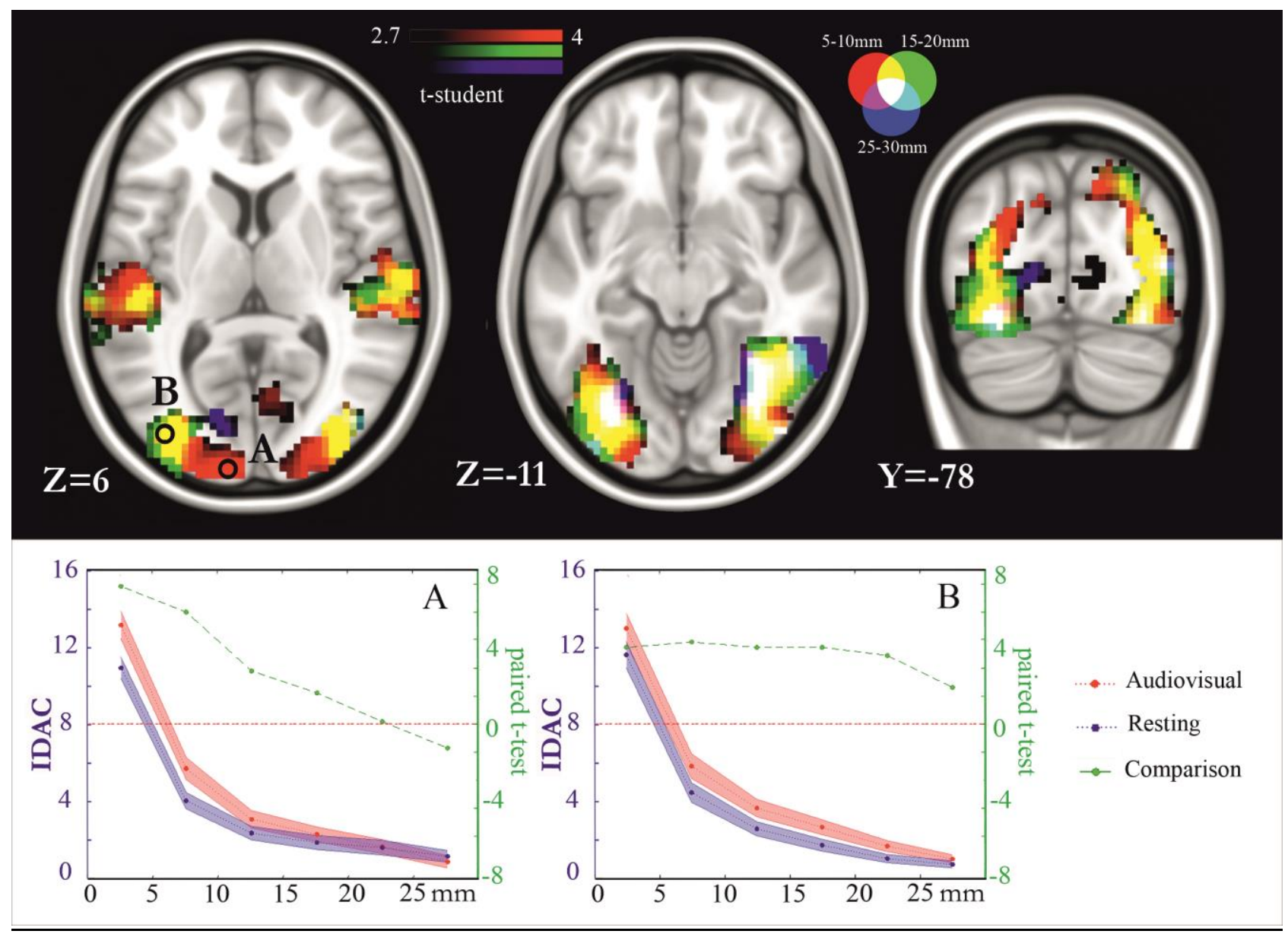

Figure 6. MULTI-DISTANCE t-MAP COMPARING STIMULATION > RESTING.

TOP: Multi-distance paired t-map comparing IDAC values from AUDIO-VISUAL STIMULATION > REST, calculated at three different distance lags. Being a post-hoc analysis, results were masked with significant clusters from the paired Hotelling's $T^{2}$ maps (see Figure 5). Visual and auditory cortices show significant increases in functional connectivity within a short neighborhood, which is indicated by red and yellow (yellow combines red and green colors); extrastriate cortex and parts of the fusiform gyrus tend to present differences at all distances (white). BOTTOM: IDAC curves with their corresponding paired t-test profile plots from ROIs A and B indicated on the map above. Coordinates in Montreal Neurological Institute (MNI) space. 

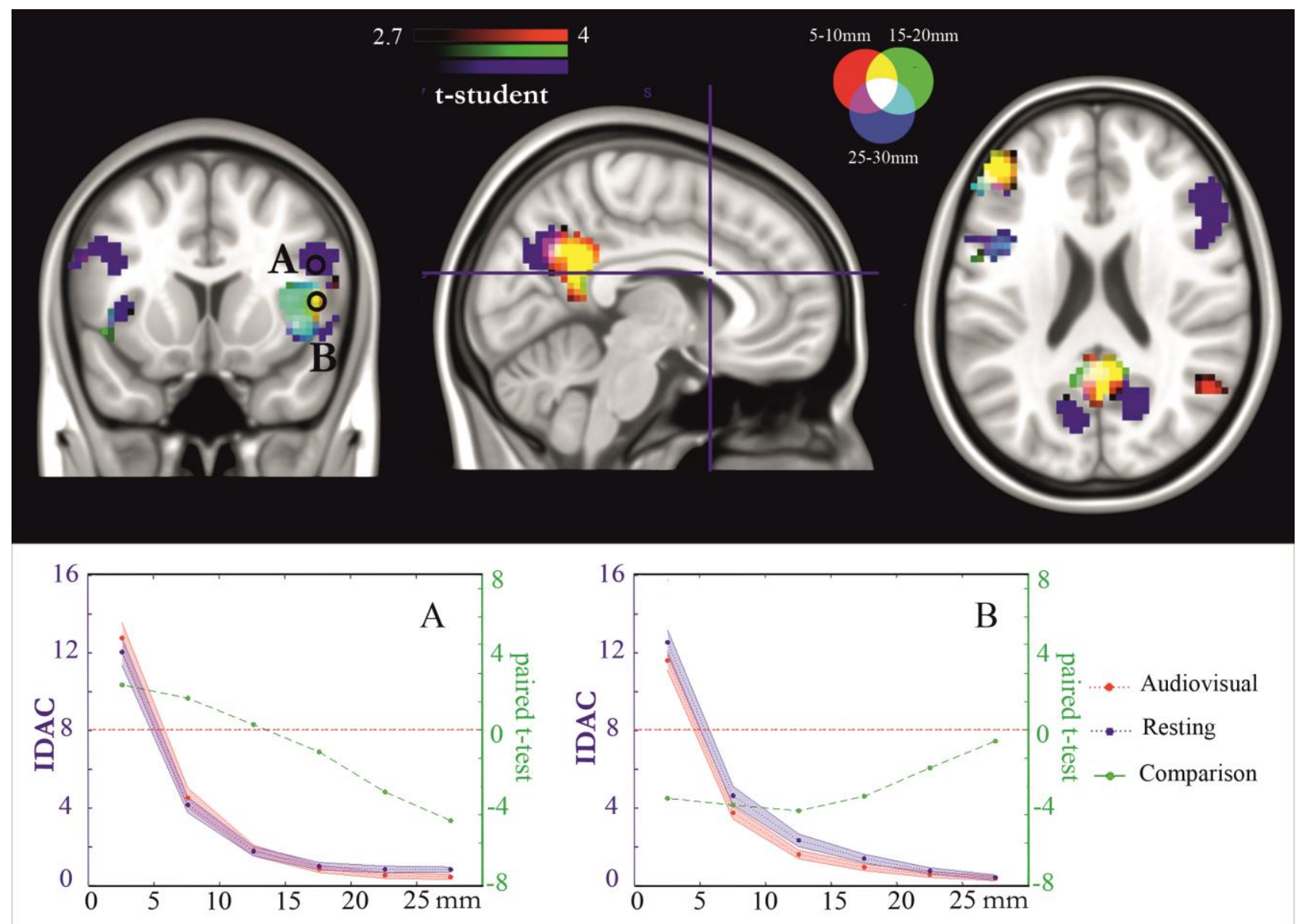

Figure 7. MULTI-DISTANCE t-MAP COMPARING STIMULATION < RESTING.

TOP: Multi-distance paired t-map comparing STIMULATION < RESTING of IDAC values calculated at three different distances. Being a post-hoc analysis, results were masked with significant clusters from the paired Hotelling's $\mathrm{T}^{2}$ maps (see Figure 5). BOTTOM: IDAC curves with their corresponding paired t-test profile plots from ROIs A and B indicated on the map above. The curves allow us to interpret why RGB coloring is important. Blue areas correspond to significant lower connectivity at the farthest local peripheries (A) whereas yellow correspond to short and medium local peripheries (B). 
Table 1. Comparison of different brain functional states.

\begin{tabular}{lcccc}
\hline \multicolumn{1}{c}{ Cluster location } & $\begin{array}{c}\text { Cluster size } \\
\text { (voxels) }\end{array}$ & $\begin{array}{c}\text { Cluster size } \\
\text { corrected } \\
\text { p-value }\end{array}$ & $\begin{array}{c}\text { Peak } \\
\text { Hotelling's T }\end{array}$ & $\begin{array}{c}\text { MNI } \\
\text { Coordinates }\end{array}$ \\
\hline Visual Cortex & 4065 & $<1.0 \mathrm{e}-06$ & 24.02 & $-32,-84,13$ \\
PCC \& Precuneus & $4065^{*}$ & $<1.0 \mathrm{e}-06$ & 14.3 & $-2,-52,28$ \\
Right Lateral Frontal Cortex & 747 & $<1.0 \mathrm{e}-06$ & 12.1 & $46,9,27$ \\
Right Anterior Insula & $747^{*}$ & $<1.0 \mathrm{e}-06$ & 11.6 & $42,13,0$ \\
Left Lateral Frontal Cortex & 697 & $<1.0 \mathrm{e}-06$ & 10.5 & $-45,40,20$ \\
Left Anterior Insula & $697 *$ & $<1.0 \mathrm{e}-06$ & 15.7 & $-45,5,18$ \\
Left Auditory Cortex & 364 & $<1.0 \mathrm{e}-06$ & 12.05 & $-50,-18,6$ \\
Right Auditory Cortex & 249 & $<1.0 \mathrm{e}-06$ & 8.73 & $68,-22,6$ \\
Left Supramarginal and Parietal & 303 & $<1.0 \mathrm{e}-06$ & 7.6 & $-30,-58,48$ \\
Cortex & & $<0.001$ & 8.5 & $55,-32,44$ \\
Right Supramarginal Cortex & 94 & $<1.0 \mathrm{e}-06$ & 7.3 & $-12,30,-18$ \\
Left Orbito-Frontal Cortex & 189 & $<0.05$ & 5.6 & $9,54,-18$ \\
Right Orbito-Frontal Cortex & 53 & $<0.0001$ & 7.8 & $49,-54,21$ \\
Right Angular Cortex & 146 & $<0.02$ & 8.5 & $-52,-59,21$ \\
Left Angular Cortex & 68 & $<0.05$ & 5.8 & $7,4,52$ \\
Right Anterior Cingulate Cortex & 59 & $<0.005$ & 7.9 & $-19,41,33$ \\
Left Superior Frontal Cortex & 70 & & & \\
\hline
\end{tabular}

x y z, coordinates $(\mathrm{mm})$ given in Montreal Neurological Institute (MNI) space. Statistics at corrected threshold $\mathrm{P}_{\mathrm{FWE}}<0.05$ estimated using Monte Carlo simulations. *Same cluster. PCC, posterior cingulate cortex. 